\title{
Experimental Investigation of Trapped Sine-Gordon Solitons
}

\author{
Davidson, A.; Dueholm, B.; Kryger, B.; Pedersen, Niels Falsig
}

Published in:

Physical Review Letters

Link to article, DOI:

10.1103/PhysRevLett.55.2059

Publication date:

1985

Document Version

Publisher's PDF, also known as Version of record

Link back to DTU Orbit

Citation (APA):

Davidson, A., Dueholm, B., Kryger, B., \& Pedersen, N. F. (1985). Experimental Investigation of Trapped SineGordon Solitons. Physical Review Letters, 55(19), 2059-2062. https://doi.org/10.1103/PhysRevLett.55.2059

\section{General rights}

Copyright and moral rights for the publications made accessible in the public portal are retained by the authors and/or other copyright owners and it is a condition of accessing publications that users recognise and abide by the legal requirements associated with these rights.

- Users may download and print one copy of any publication from the public portal for the purpose of private study or research.

- You may not further distribute the material or use it for any profit-making activity or commercial gain

- You may freely distribute the URL identifying the publication in the public portal 


\title{
Experimental Investigation of Trapped Sine-Gordon Solitons
}

\author{
A. Davidson, (a) B. Dueholm, B. Kryger, and N. F. Pedersen \\ Physics Laboratory I, The Technical University of Denmark, DK-2800 Lyngby, Denmark \\ (Received 7 August 1985)
}

\begin{abstract}
We have observed for the first time a single sine-Gordon soliton trapped in an annular Josephson junction. This system offers a unique possibility to study undisturbed soliton motion. In the context of perturbation theory, the soliton may be viewed as a relativistic particle moving under a uniform force with damping. Accordingly our experimental results are displayed directly in a forceversus-momentum plane, where they may be described by a theoretically derived universal curve.
\end{abstract}

PACS numbers: $74.50 .+\mathrm{r}$

Sine-Gordon solitons play a unique role in physics as one of the clearest examples of nonlinear wave motion. In solid-state physics appeals to the sineGordon system are made from such diverse fields as thermodynamics, topological excitations, magnetism, and Josephson tunneling. Undoubtedly the physical system with the closest correspondence to a pure sineGordon situation is the long (one-dimensional) Josephson transmission line where detailed experimental confirmation of sine-Gordon soliton dynamics has been obtained. ${ }^{1}$ In the Josephson junction, the sine-Gordon soliton is a superconducting fluxoid quantum (a fluxon) that threads the tunnel barrier and is of magnitude $\phi_{0}=2.07 \times 10^{-15} \mathrm{~Wb}$. Because of the usual topology of tunnel junctions, however, previous experimental data have been obtained from solitons that suffer collisions with other solitons and/or the ends of the line. This fact complicates much of the data interpretation, and has left many questions open.

In the usual model, ${ }^{2}$ soliton motion is assumed to be governed by the perturbed sine-Gordon equation,

$$
-\phi_{x x}+\phi_{t t}+\sin \phi=-\alpha \phi_{t}+\beta \phi_{x x t}+\eta(x) .
$$

With introduction of the soliton momentum, $p$ $=\int \phi_{x} \phi_{t} d x$, perturbation analysis yields a relativistic equation of motion for a soliton particle, ${ }^{2-5}$

$$
\frac{d p}{d t}=-\alpha p\left[1+\frac{\beta}{3 \alpha}\left(1+p^{2}\right)\right]+\frac{\pi \eta}{4} .
$$

Hence, the steady-state relation between force $\eta$ and momentum is given by

$$
\eta=\frac{4 \alpha p}{\pi}\left[1+\frac{\beta}{3 \alpha}\left(1+p^{2}\right)\right], \quad p=u \gamma(u) .
$$

$u$ is the normalized soliton velocity $u=v / c$ where $c$ is the speed of light in the junction. The Lorentz factor is then $\gamma(u)=1 /\left(1-u^{2}\right)^{1 / 2}$. The momentum $p$ has been normalized to $m_{0} c$, where $m_{0}$ is the rest mass of the soliton given by $m_{0}=8 \phi_{0} J \lambda_{j} W / 2 \pi c^{2}$, where $W$ is the junction width. Typically $m_{0}$ is the order of one electron mass. In all the above equations $\phi$ is the superconducting phase difference, $\alpha=G(h /$ $\left.4 \pi e d \mu_{0} J\right)^{1 / 2}$ is the tunneling loss parameter, and $\beta$ the surface loss. ${ }^{2,6} \eta=I / I_{0}$ is the bias current, assumed to be uniformly distributed, and normalized to the total critical current $I_{0}$. The spatial variable is measured in units of $\lambda_{j}=1 /\left(h / 4 \pi e d \mu_{0} J\right)^{1 / 2}$, the Josephson penetration depth, and time in units of $1 / \omega_{0}$ $=1 /(4 \pi e J / h C)^{1 / 2}$, the reciprocal angular plasma frequency. Here $d$ is the magnetic thickness of the tunnel barrier, $J$ the Josephson current density, and $C$ and $G$ the capacitance and tunnel conductance per unit area, respectively. In this system of normalization the voltage across the junction is in units of $h \omega_{0} / 4 \pi e$, and is related to the velocity $u$ of the soliton by $V=2 \pi u / l$, where $l$ is the normalized length of the junction.

In Eqs. (2) and (3) there is no account of either boundary collisions or soliton-antisoliton interactions. For real junctions this has been a severe restriction, since for the usual geometries both boundary collisions and soliton-antisoliton collisions necessarily occur, and may not be neglected ab initio. ${ }^{7}$

We report here on an annular, or ring-shaped, Josephson tunnel junction, Fig. 1, to which the simple

(a)

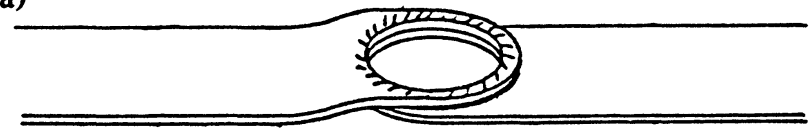

(b)

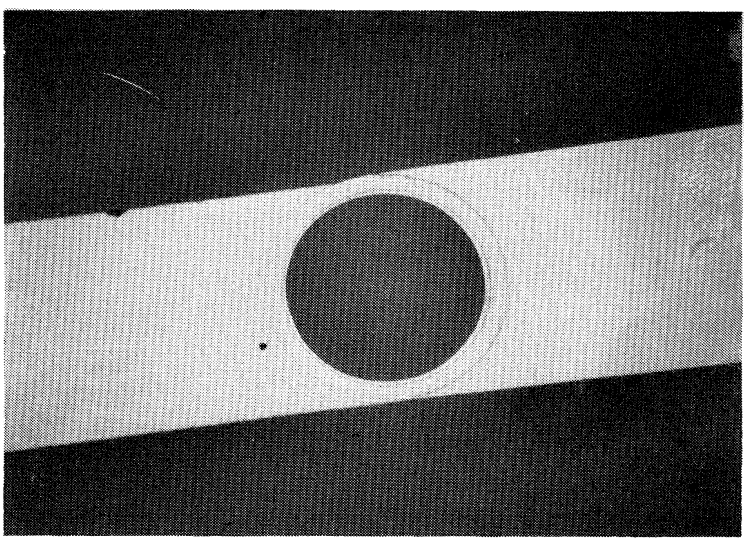

FIG. 1. (a) A schematic of the annular junction that has successfully trapped a single sine-Gordon soliton. (b) An optical micrograph of a typical junction as fabricated, where the overall diameter of the junction is about $300 \mu \mathrm{m}$. 
perturbation theory is expected to apply. Inherently this geometry has no boundaries and hence boundary reflections are ruled out. As a result of fluxoid quantization in a superconducting ring, one or more solitons may be trapped in the junction upon cooling through the transition temperature. If no solitons are trapped while cooling through $T_{c}$, then soliton-antisoliton pairs may be created by cycling around the hysteretic $I-V$ curve. Hence, starting from zero trapped solitons the annular junction is a unique system for studying soliton-antisoliton collisions unmasked by boundary effects. If only one soliton is trapped it will suffer neither boundary reflections nor collisions with other solitons, and it is possible to study undisturbed soliton motion.

With the annular junction shown in Fig. 1, we have observed such a single soliton. The sample was a planar $\mathrm{Nb}$-oxide- $\mathrm{Pb}$ junction with a current density of about $60 \mathrm{~A} / \mathrm{cm}^{2}$, a physical length $L$ (circumference) of about $0.9 \mathrm{~mm}$, and a normalized length $l$ of $15 . \lambda_{j}$ was about $60 \mu \mathrm{m}$, and the width $W$ was about $20 \mu \mathrm{m}$. The base and counter electrode films were both about $250 \mathrm{~nm}$ thick. Figure 2 (dashed curves) shows clear evidence that soliton trapping has occurred, in this case a single soliton. If we neglect the interactions between solitons and surface losses, Eq. (3) predicts steps at voltages given by

$$
V_{n}=\frac{2 \pi n / l}{\left[1+(4 \alpha / \pi \eta)^{2}\right]^{1 / 2}}
$$

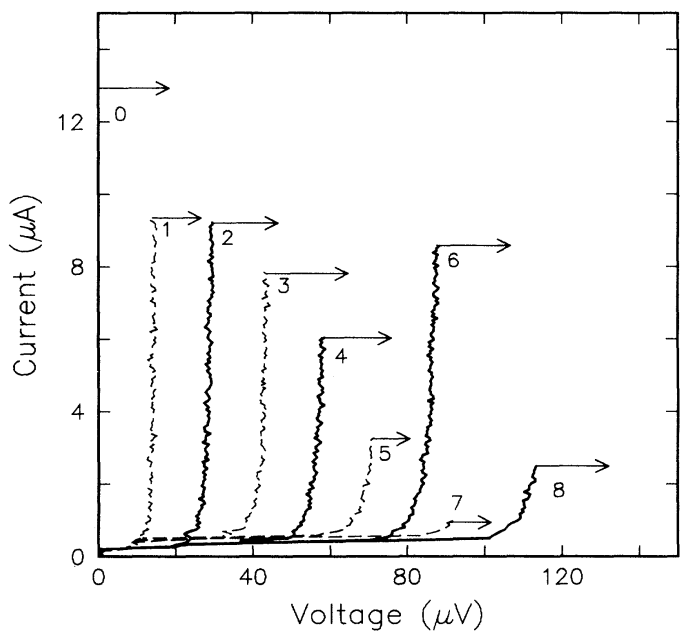

FIG. 2. Current vs voltage for various numbers of solitons at $4.2 \mathrm{~K}$. Full curves: no solitons trapped. Dashed curves: one trapped soliton. The number next to each curve is the total number of solitons and antisolitons moving to produce that curve. The right-pointing arrows at the top of each curve mark the points at which the junction switches out of the various soliton branches. Junction parameters are $J=67 \mathrm{~A} / \mathrm{cm}^{2}, \quad \lambda_{j}=59 \mu \mathrm{m}, L=875 \mu \mathrm{m}, L / \lambda_{j}=14.8$, $\alpha=0.018$, and $\beta=0.01$. where $n$ is the total number of solitons and antisolitons. WIth one soliton trapped the odd series $n=1,3,5, \ldots$ is expected, since additional solitons can be created only in soliton-antisoliton pairs. This is exactly the situation for the dashed lines in Fig. 2. We note that this state is extremely stable and persists even after the voltage has been cycled through the hysteresis loop to the gap and back. This odd series of steps is obtained in the experiments by cooling quickly through $T_{c}$ in the presence of a bias current through the junction. In the zero trapped soliton case, obtained by heating and carefully cooling through $T_{c}$ with zero bias, the even series $n=0,2,4,6, \ldots$ is observed (full curves in Fig. 2).

We always observed the full critical current in the even series, as expected. However, the odd series, which ideally should have zero critical current, was experimentally associated with a small (8\%) critical current. This small critical current was observed to be double valued, depending on the history of the junction. From this we infer the existence of two pinning sites (possibly related to the geometry) of slightly different strengths on the ring. With the soliton initially at rest in one of these wells a certain force (bias) is required to initiate the motion. Depending on which well trapping occurred in, one of two different critical currents will be measured. A difference in slopes on the two sides of the wells could account for a small asymmetry in the critical currents for positive and negative dc bias (not shown in the figure).

In previous numerical simulations ${ }^{8}$ of the annular junction some predictions of the perturbation theory have been confirmed, but experiments ${ }^{9}$ have been only partially successful. The annular junctions used in Ref. 9 seemed unable to trap a single soliton, although soliton-antisoliton pairs were observed. Since that junction and the one reported on here have similar normalized parameters (including film thicknesses), we must conclude that proper conditions for trapping a soliton in the junction are not known. We also call attention to the fact that the annular junction reported on here and that of Ref. 9 both have remarkably uniform bias-current distributions. This is inferred from the ratio of the critical current to the current step at the gap voltage. This ratio is about 0.5 , which is close to its value for small area junctions made of the same materials.

Figure 3 shows the experimental results for the force versus momentum for a single soliton trapped in the new geometry junction, and measured at two diffrerent temperatures, corresponding to critical currents of 12.7 and $3.46 \mathrm{~mA}$. The $I-V$ curve was measured with a low-noise room-temperature preamplifier and recorded by a microcomputer with a precise analogto-digital converter. For a comparison to Eq. (3) we must know the critical current $I_{0}$ without trapped soli- 


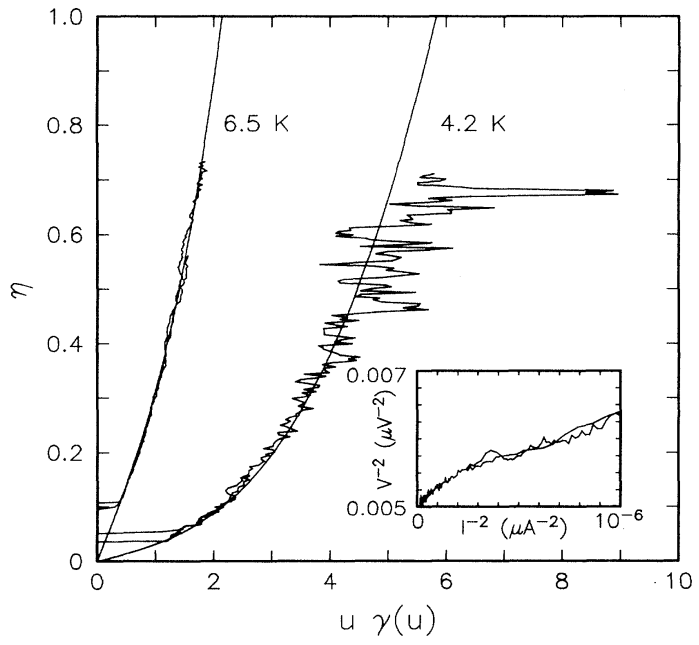

FIG. 3. Force $(\eta)$ vs momentum $[u \gamma(u)]$ for the $n=1$ step (a single trapped soliton) for two temperatures. The curve at the left is for a temperature just below $T_{c}$, about 6.5 $K$. It was fitted as described in the text with $\beta=0.1$, $\alpha=0.18, V_{c}=12.51 \mu \mathrm{V}$, and $I_{0}=3.46 \mathrm{~mA}$. The curve at the right was taken at about $4.2 \mathrm{~K}$ and fitted with $\beta=0.01$, $\alpha=0.018, V_{c}=14.38 \mu \mathrm{V}$, and $I_{0}=12.7 \mathrm{~mA}$.

tons (to compute $\eta$ ), and also the voltage $V_{c}$ corresponding to soliton motion at the speed of light in the barrier [to compute $\gamma(u)$ ]. The junction's critical current was measured independently at different temperatures before attempting to trap a soliton. We obtained good estimates of $V_{c}$ at different temperatures by plotting the computer-stored $I-V$ curves as $V^{-2}$ against $I^{-2}$ and extrapolating to the $V^{-2}$ axis intercept. Such a plot would be a straight line if the $\beta$ damping term were negligible, according to Eq. (4). An example of this kind of graph is the inset in Fig. 3. Using $V_{c}$ found in this way, and calculating $u=V / V_{c}$, we could obtain good fits to Eq. (3) over our entire temperature range. Furthermore, we found that we could improve the fits at high momentum by correcting the estimates of $V_{c}$ upward by about $1 \%$, and these corrected values were used in preparing Fig. 3. To our knowledge Fig. 3 shows the first experimentally obtained force-versus-momentum curves for a relativistic particle in a solid-state system. An important result for our junction is that $\beta / \alpha$ is very accurately temperature independent: Exactly the same ratio was used for fitting the data at all temperatures. Since both tunneling losses $(\alpha)$ and skin losses $(\beta)$ depend on the density of quasiparticles, this result is reasonable.

For the highest temperature $(\alpha=0.18)$, we see from Fig. 3 that the linear term in Eq. (3) is dominant. For the lower temperature $(\alpha=0.018)$ the momentum becomes larger and the effect of the cubic term is dramatic, leaving no doubt about the existence of the skin-damping term. We may be certain that the cubic

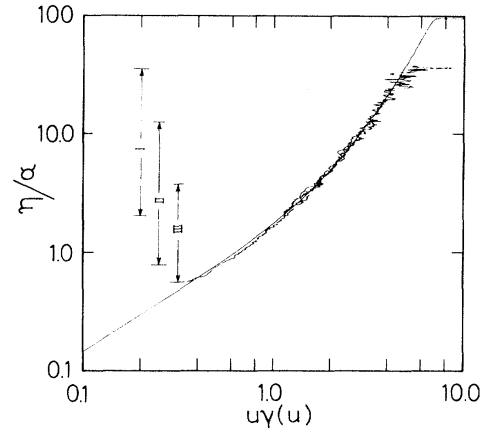

FIG. 4. The universal force-momentum curve for this junction (smooth line) for $\beta / \alpha=0.556$. The rough lines are data taken with a single soliton trapped in the junction (on the $n=1$ step) at three different temperatures, all fitted with the constant $\beta / \alpha$. Notice that the force $(\eta)$ has been scaled with $\alpha$. The three Roman numerals show the three ranges of $\eta / \alpha$ covered by the three temperatures from $4.2 \mathrm{~K}$, region I, to some intermediate temperature, II, to the highest temperature, about $6.5 \mathrm{~K}$, region III.

dependence in our data is from skin damping and not from a cubic part in the quasiparticle damping since for the latter case $\beta / \alpha$ would not be temperature independent. Except for the small supercurrent the agreement between Eq. (3) and the experiment is very satisfactory. We also note from Fig. 3 that the experimental curves terminate near $\eta=0.7$. We speculate that this permature switching may be related to the type of correction to Eq. (3) (generalized to $\beta \neq 0$ ) first suggested by Buettiker and Landauer. ${ }^{3}$

Assuming for a given junction that $\beta / \alpha$ is independent of temperature, we note from Eq. (3) that $\eta / \alpha$ is a function of momentum only. This universal curve is shown in Fig. 4 in a log-log plot together with the experimental points at three different temperatures.

Finally, we note that we could observe fine structure in the form of small steps, sometimes hysteretic, on the $n=1 I-V$ curve. Earlier experiments and simulations suggest that this type of structure is due to frequency locking between the soliton and Josephson plasma oscillations caused by collisions and reflections of solitons. That this fine structure sitll exists for a soliton with neither collisions nor boundaries invites further research, but we suspect that it arises from soliton interaction with the two trapping centers.

In summary, we have successfully trapped a single soliton in an annular Josephson junction. The forceversus-momentum curve corresponding to the $n=1$ step was described very well by a universal curve derived from perturbation theory, and consistent with a relativistic particle picture. Also, we have experimentally demonstrated the existence and influence of surface losses and have shown how this modifies the force-versus-momentum curve.

This work has been supported in part by the Danish 
Natural Science Research Council, and by a joint study agreement between the Technical University of Denmark and IBM Research, Yorktown Heights, New York. One of us (A.D.) wishes to thank The Technical University of Denmark for hospitality and support during the course of this work.

(a) Permanent address: IBM Research, Yorktown Heights, N. Y. 10598.

${ }^{1}$ A. Barone and G. Paterno, Physics and Applications of the Josephson Effect (Wiley-Interscience, New York, 1982).

2D. W. McLaughlin and A. C. Scott, Phys. Rev. A 18, 1652 (1978).

${ }^{3} \mathrm{M}$. Buettiker and R. Landauer, Nonlinear Phenomena at Phase Transitions and Instabilities (Plenum, New York,
1982), p. 111.

${ }^{4}$ G. Reinisch and J. C. Fernandez, Phys. Rev. B 25, 7352 (1982).

${ }^{5}$ D. J. Bergman, E. Ben-Jacob, Y. Imry, and K. Maki, Phys. Rev. A 27, 3345 (1983).

6 W. J. Johnson, Ph.D. thesis, University of Wisconsin 1968 (unpublished).

${ }^{7}$ N. F. Pedersen, M. R. Samuelsen, and D. Welner, Phys. Rev. B 30, 4057 (1984).

${ }^{8}$ A. Davidson and N. F. Pedersen, Appl. Phys. Lett. 44, 465 (1984).

${ }^{9}$ B. Dueholm, A. Davidson, C. C. Tsuei, M. J. Brady, K. H. Brown, A. C. Callegari, M. M. Chen, J. H. Greiner, H. C. Jones, K. K. Kim, A. W. Kleinsasser, H. A. Notarys, G. Proto, R. H. Wang, and T. Yogi, in Proceedings of the Seventeenth International Conference on Low Temperature Physics, edited by U. Eckern et al. (North-Holland, Amsterdam, 1984), p. 691. 
(a)

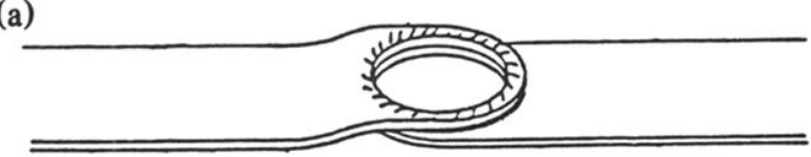

(b)

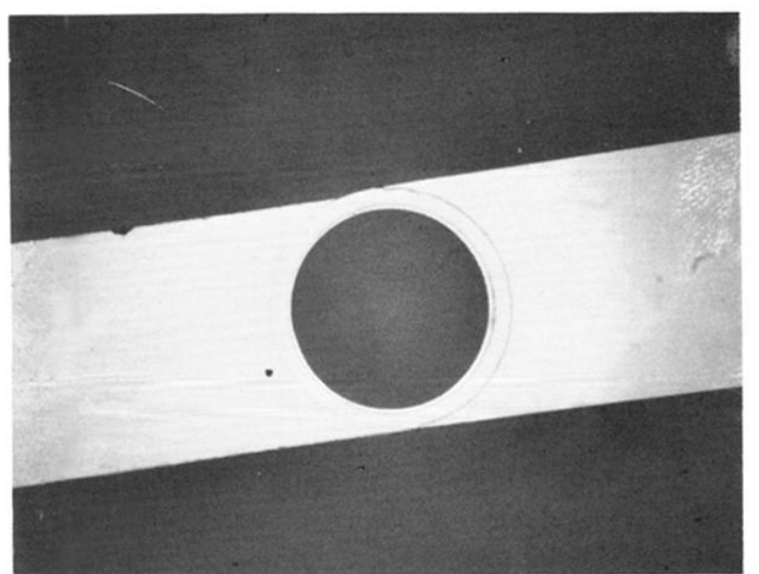

FIG. 1. (a) A schematic of the annular junction that has successfully trapped a single sine-Gordon soliton. (b) An optical micrograph of a typical junction as fabricated, where the overall diameter of the junction is about $300 \mu \mathrm{m}$. 